\title{
PpMYB36 Encodes a MYB-Type Transcription Factor That Is Involved in Russet Skin Coloration in Pear (Pyrus pyrifolia)
}

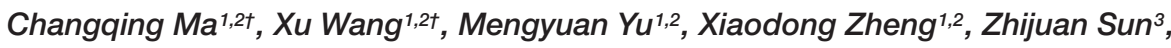 \\ Xiaoli Liu ${ }^{1,2}$, Yike Tian ${ }^{1,2}$ and Caihong Wang ${ }^{1,2 *}$ \\ ${ }^{1}$ College of Horticulture, Qingdao Agricultural University, Qingdao, China, ${ }^{2}$ Engineering Laboratory of Genetic Improvement \\ of Horticultural Crops of Shandong Province, Qingdao, China, ${ }^{3}$ College of Life Science, Qingdao Agricultural University, \\ Qingdao, China
}

\section{OPEN ACCESS}

Edited by:

Pavlina Drogoudi, Hellenic Agricultural Organisation, Greece

Reviewed by:

Gunars Lacis,

Institute of Horticulture (LatHort),

Latvia

Carolina Font i Forcada, Institute of Agrifood Research and Technology (IRTA), Spain

*Correspondence: Caihong Wang chwang6068@163.com

tThese authors have contributed equally to this work

Specialty section:

This article was submitted to

Plant Breeding,

a section of the journal

Frontiers in Plant Science

Received: 14 September 2021

Accepted: 13 October 2021

Published: 08 November 2021

Citation:

Ma C, Wang X, Yu M, Zheng $X$, Sun Z, Liu X, Tian Y and Wang $C$

(2021) PpMYB36 Encodes a MYB-Type Transcription Factor That Is Involved in Russet Skin Coloration

in Pear (Pyrus pyrifolia).

Front. Plant Sci. 12:776816. doi: 10.3389/fp/s.2021.776816
Fruit color is one of the most important external qualities of pear (Pyrus pyrifolia) fruits. However, the mechanisms that control russet skin coloration in pear have not been well characterized. Here, we explored the molecular mechanisms that determine the russet skin trait in pear using the $F_{1}$ population derived from a cross between russet skin ('Niitaka') and non-russet skin ('Dangshansu') cultivars. Pigment measurements indicated that the lignin content in the skin of the russet pear fruits was greater than that in the non-russet pear skin. Genetic analysis revealed that the phenotype of the russet skin pear is associated with an allele of the PpRus gene. Using bulked segregant analysis combined with the genome sequencing (BSA-seq), we identified two simple sequence repeat (SSR) marker loci linked with the russet-colored skin trait in pear. Linkage analysis showed that the PpRus locus maps to the scaffold NW_008988489.1: 53297-211921 on chromosome 8 in the pear genome. In the mapped region, the expression level of LOC103929640 was significantly increased in the russet skin pear and showed a correlation with the increase of lignin content during the ripening period. Genotyping results demonstrated that LOC103929640 encoding the transcription factor MYB36 is the causal gene for the russet skin trait in pear. Particularly, a W-box insertion at the PpMYB36 promoter of russet skin pears is essential for PpMYB36-mediated regulation of lignin accumulation and russet coloration in pear. Overall, these results show that PpMYB36 is involved in the regulation of russet skin trait in pear.

Keywords: Pyrus pyrifolia, russet skin, lignin biosynthesis, SSR markers, PpMYB36

\section{INTRODUCTION}

Pear (Pyrus pyrifolia) is highly valued as a cultivated fruit crop around the world. Fruit color is one of the most vital external qualities of pear that determines market acceptance by consumers (Ma et al., 2018a). The color of pear fruit skin can be divided into two types: a ground color that includes green and yellow, and a cover color with russet (red-brown) and red colors (Heng et al., 2016). Different pear cultivars have distinct coloration, which can result from genetic or environmental factors (Heng et al., 2014). Previous studies on pear coloration have mainly explored the red skin 
which depends on anthocyanin biosynthesis (Wang et al., 2017; Liu et al., 2019). In addition, the russet skin is an important trait that protects pear fruits from environmental stresses caused by diseases, insects, and unfavorable weather, as well as shipping (Inoue et al., 2006). Therefore, exploring and using the genetic resources for russet skin is critical for progress in pear breeding.

Transcriptomic and proteomic approaches have been used to explore genes responsible for russet skin color in pears (Legay et al., 2015; Shi et al., 2019a). In particular, the russet skin is related to the biosynthesis of lignin, and is regulated in vivo by many structural and regulatory genes (Wang Y. Z. et al., 2016; Shi et al., 2019b). The structural genes that are directly involved in the biosynthesis of lignins encode phenylalanine ammonia-lyase $(P A L)$, cinnamate 4-hydroxylase $(C 4 H)$, 4-coumarate-CoA ligase $(4 C L)$, shikimate/quinate hydroxycinnamoyl transferase $(\mathrm{HCT})$, coumarate 3-hydroxylase $(\mathrm{C} 3 \mathrm{H})$, cinnamoyl CoA reductase $(C C R)$, cinnamyl alcohol dehydrogenase $(C A D)$, caffeoyl CoA O-methyltransferase (CCoAOMT), and caffeic acid/5-hydroxyferulic acid O-methyltransferase (COMT) (Lam et al., 2017; Liu et al., 2018). Transcription factors in the R2R3-MYB family regulate the lignin biosynthesis pathway in plants (Ohtani and Demura, 2019; Geng et al., 2020). AtMYBs specifically activate lignin biosynthesis genes, which control defense-induced lignification and basal immunity in Arabidopsis thaliana (Chezem et al., 2017). CsMYBs were also found to regulate fruit juice sac lignification through fine-tuning of the expression of Cs4CL in orange (Citrus sinensis) (Jia et al., 2018). However, the contribution of MYB family members to russet skin coloration in pear is unclear.

In many horticultural fruit species, marker-assisted selection (MAS) for major agricultural traits has been developed. For example, molecular markers associated with pear scab resistance, harvest time, and dwarf tree architecture have been developed and applied to pear breeding programs (Terakami et al., 2006; Yamamoto et al., 2014; Wang C. H. et al., 2016). In our previous study, the gene that determines fruit russet skin was localized to linkage group 8 (LG8) of the pear consensus genetic map using simple sequence repeat (SSR) markers (Song et al., 2010). However, no candidate genes have been identified that control the pear russet skin trait on LG8. Rapid advances in DNA sequencing of the pear genome and pear haploid cell genotyping technology have provided invaluable new resources for genetics and biological research (Wu et al., 2013; Shi et al., 2019c). Recently, bulked segregant analysis combined with genome sequencing, known as BSA-seq, has proven successful for rapidly mapping genes in several vegetable and fruit species (Huo et al., 2016; Dougherty et al., 2018). Using BSA-seq, the ABA1/ZEP gene for thermal tolerance was efficiently identified in lettuce (Lactuca sativa), four major genome-wide quantitative trait loci responsible for fruit acidity were mapped on chromosomes 8 and 16 of apple (Malus domestica), and CcPRR2 (PSEUDORESPONSE REGULATOR 2) was identified as a candidate gene for the control of fruit color in pepper (Capsicum chinense) (Huo et al., 2016; Jia et al., 2018; Lee et al., 2020).

In the present study, we aimed to elucidate the molecular mechanisms that determine the russet skin trait in pear. SSR markers were identified and mapped in the pear genome based on gene location using BSA-seq analysis. This research enhances our understanding of the molecular mechanisms underpinning russet skin coloration in pear.

\section{MATERIALS AND METHODS}

\section{Plant Materials}

An $\mathrm{F}_{1}$ population of 150 individuals was derived from crossing 'Niitaka' (NTK, russet skin pear cultivar) with 'Dangshansu' (DSS, non-russet skin pear cultivar). The trees were 17 years old and were planted at a density of $2.5 \times 0.5 \mathrm{~m}$ at the Fruit Research Station of Qingdao Agricultural University (Laiyang, Shandong Province, China). Ripening fruit samples were collected at 25, 50, 75,100 , and 125 days after full bloom (DAFB) for lignin and chlorophyll measurements and gene expression analysis. Each sample consisted of 12 fruits, and three biological replicates were harvested per time point. Young leaf samples were collected from each tree in the spring. Fruit peel was collected with a peeler, immediately frozen in liquid nitrogen, and stored at $-80^{\circ} \mathrm{C}$ prior to its use in the experiments.

Mature 'Korla' pear fruits were used for infection of transgenic analysis according to Bai et al. (2019). Pear calli were induced from the flesh of young 'Clapp's Favorite' ( $P$. communis) fruits on NN69 (NITSCH and NITSCH 1969) solid medium. The first-generation calli were subcultured several times, and the rapidly growing soft calli were screened and maintained on Murashige-Skoog (MS) solid medium in the dark according to the protocol of Bai et al. (2019). Nicotiana benthamiana plants were grown in vitro at $25^{\circ} \mathrm{C}$ on solid MS medium (Murashige and Skoog, 1962).

\section{Chlorophyll and Lignin Measurements}

Measurement of total chlorophyll content was performed as described previously (Lichtenthaler and Wellburn, 1983). In brief, pear skin tissue $(0.5 \mathrm{~g})$ was homogenized in $5 \mathrm{~mL}$ of $80 \%$ acetone and then left in the dark for $24 \mathrm{~h}$. After centrifugation for $20 \mathrm{~min}$ at $13,000 \times \mathrm{g}$, the absorbance of the supernatant was measured at 663 and $645 \mathrm{~nm}$ using a UV-2550 ultraviolet spectrophotometer (Shimadzu Corp., Kyoto, Japan). The chlorophyll concentration was calculated according to the protocol of Ma et al. (2018a). Total lignin was extracted from pear skin using the Lignin Content Determination Kit (Geruisi, Suzhou, Jiangsu Province, China). Three independent biological replicates were performed for each experiment.

\section{DNA Extraction and BSA-Seq}

Leaf tissues ( $0.5 \mathrm{~g}$ each) of $\mathrm{F}_{1}$ plants with extreme phenotypic traits (russet or non-russet fruit skin) were ground to a powder in liquid nitrogen. DNA was isolated using the cetyltrimethylammonium bromide (CTAB) method (Ma et al., 2018a). DNA quality and concentration were assessed by electrophoresis on a $1 \%(\mathrm{w} / \mathrm{v})$ agarose gel and an ultramicro spectrophotometer (Thermo Fisher, Wilmington, DE, United States), respectively. A total of 50 individual plants, 25 with russet skin and 25 with non-russet skin, were chosen from the NTK $\times$ DSS $F_{1}$ population for BSA-seq analysis. 
Two pools of genomic DNA from plants with the two extreme fruit skin phenotypic coloration traits were used to construct the segregant bulks. Each parental or bulked DNA sample was sequenced to $>30 \times$ genome size depth using a paired-end 150 base strategy (Illumina $\times 10$, Illumina). ${ }^{1}$ After quality filtering, clean reads were mapped to the pear genome ${ }^{2}$ using BurrowsWheeler alignment software (Li and Durbin, 2009). SAMtools was used to obtain the read depth of the genome (Li et al., 2009). A modified G' value method was used for the statistical analysis of allelic variations between the two bulks (Magwene et al., 2011). Venn diagrams of variants identified in different samples were constructed according to Imerovski et al. (2019). Kyoto Encyclopedia of Genes and Genomes (KEGG) pathway enrichment analysis was used to identify biological processes and functions enriched for genes with variants (Xu et al., 2021).

\section{Simple Sequence Repeat Marker Detection}

Based on the BSA-seq results, SSRs were screened using the SSRIT website. ${ }^{3}$ Primers for SSR markers were designed with Primer premier 5.0 (PREMIER Biosoft International, Inc., Palo Alto, CA, United States) (Supplementary Table 3). Polymerase chain reaction (PCR) amplification assays were performed in $20 \mu \mathrm{L}$ volumes containing $60 \mathrm{ng}$ of genomic DNA, $10 \mu \mathrm{L} 2 \times$ Taq Plus Master Mix II (Vazyme, Nanjing, Jiangsu Province, China), and $0.25 \mu \mathrm{M}$ SSR primer. Reactions were subjected to an initial denaturation at $95^{\circ} \mathrm{C}$ for $5 \mathrm{~min}$, with 35 cycles of $94^{\circ} \mathrm{C}$ for $30 \mathrm{~s}$, $58^{\circ} \mathrm{C}$ for $30 \mathrm{~s}$, and $72^{\circ} \mathrm{C}$ for $60 \mathrm{~s}$ followed by a final extension step at $72^{\circ} \mathrm{C}$ for $10 \mathrm{~min}$. Amplifications were performed in a $\mathrm{T} 100^{\mathrm{TM}}$ Thermal Cycler (Bio-Rad Laboratories, Hercules, CA, United States). The amplified products were separated by electrophoresis on $3.5 \%(\mathrm{w} / \mathrm{v})$ agarose gels.

\section{Linkage Map Construction}

The segregation of SSR marker loci associated with the russet skin phenotype in pear were analyzed in the $150 \mathrm{~F}_{1}$ offspring of the NTK $\times$ DSS cross. Genetic distances between each of the marker loci and the russet skin locus were calculated. JoinMap $4.0^{4}$ with the Kosambi mapping function was used for linkage analysis.

\section{Quantitative Real-Time PCR Analysis}

Total RNA was extracted from pear tissues as described previously (Ma et al., 2018b). First-strand cDNA was synthesized using the PrimeScript ${ }^{\mathrm{TM}}$ RT reagent kit (Takara, Dalian, Liaoning Province, China). LightCycler ${ }^{\circledR} 480$ SYBR Green Master (Roche, Mannheim, Germany) was used for the qRT-PCR assays with the LightCycler ${ }^{\circledR} 480$ II system (Roche, Rotkreuz, Switzerland). The Actin gene (GenBank: AB190176) was used as the internal control for gene expression normalization. Genespecific primers were designed using Primer 5 (Supplementary Table 5). Data were analyzed using the $2^{-\Delta \Delta C T}$ method (Livak and Schmittgen, 2001).

\footnotetext{
${ }^{1}$ https://www.illumina.com/

${ }^{2}$ https://www.ncbi.nlm.nih.gov/assembly/GCF_000315295.1/

${ }^{3}$ https://archive.gramene.org/db/markers/ssrtool

${ }^{4}$ https://joinmap.software.informer.com/4.0/
}

\section{PpMYB36 Genomic Sequence Cloning}

The names and sequences of primers used for amplification of the PpMYB36 genomic sequence are given in Supplementary Table 5. DNA fragments were amplified using high-fidelity DNA polymerase (Takara) using reaction conditions recommended by the manufacturer. The PCR products were purified and cloned into the PMD 19-T vector (Takara). Nucleotide sequences of 10 independent clones of each fragment per sample were determined. BLAST analysis of the amino acid sequence of PpMYB36 was performed in the Arabidopsis information resource, ${ }^{5}$ and a phylogenetic tree was constructed using MEGA 5.2 (Tamura et al., 2011). Promoter sequence analysis was performed using the PlantCARE online database. ${ }^{6}$

\section{PpMYB36 Subcellular Localization}

To determine the subcellular localization of the PpMYB36 promoter, the PpMYB36 coding region without the stop codon was subcloned into the $p M D C 83$ vector to generate a 35S::PpMYB36-GFP fusion plasmid. The plasmid was then introduced into Agrobacterium tumefaciens strain GV3101. The leaves of 5-week-old $N$. benthamiana plants were infiltrated with GV3101 harboring 35S::PpMYB36-GFP or mCherry (control). Subcellular localization was observed with a laser confocal microscope $(\times 40)$ (FV10-ASW, Olympus, Tokyo, Japan) 3 days after transformation (Zhang et al., 2019).

\section{Vector Construction and Transformation}

The coding sequence of $P$ PMYB36 was cloned and ligated into the $p B 1121$ vector in the sense and anti-sense directions to generate 35S::PpMYB36 and 35S::anti-PpMYB36, respectively. The primers used are given in Supplementary Table 6. The 35S::PpMYB36 and 35S::anti-PpMYB36 plasmids were transformed separately into A. tumefaciens strain EHA105, and the plasmid-bearing strains were then infiltrated into fruit skin using a needleless syringe. The agro-infiltrated samples were incubated overnight in the dark at room temperature, then exposed to white light $\left(540 \mu \mathrm{mol} \cdot \mathrm{m}^{-2} \cdot \mathrm{s}^{-1}\right)$ with a $16 \mathrm{~h}$ photoperiod at $25^{\circ} \mathrm{C}$ in a growth chamber as previously described (Ma et al., 2019).

\section{PpMYB36 Promoter Activity Assay}

$\beta$-Glucuronidase (GUS) and luciferase (LUC) assays were performed as previously described (Zhao et al., 2016). A. tumefaciens strain GV3101 cultures harboring the PpMYB36 promoter from russet skin pear (ProR) and non-russet skin pear (ProNR) together with the Super empty vector ( $p C A M B I A 1300)$ were co-infiltrated into pear calli. Super::LUC was added as an internal control. GUS and LUC activities were quantified after 3 days, and the GUS/LUC ratio was used for the final quantification of the relative GUS activity. To further analyze the relative activities of the different promoters on PpMYB36 expression, ProR::PpMYB36 and ProNR::PpMYB36 were cloned into $p B 1121$ to replace 35S::GUS. A. tumefaciens

\footnotetext{
${ }^{5}$ https://www.arabidopsis.org/

${ }^{6}$ http://bioinformatics.psb.ugent.be/webtools/plantcare/html/
} 

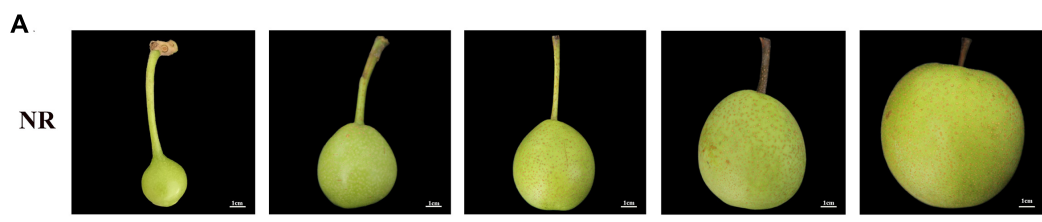

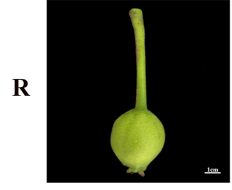

25

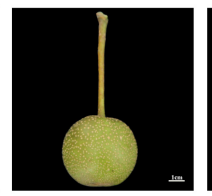

50

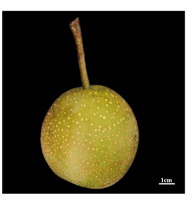

75

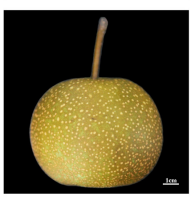

100

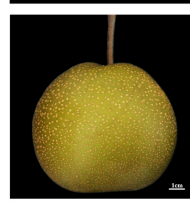

125

B

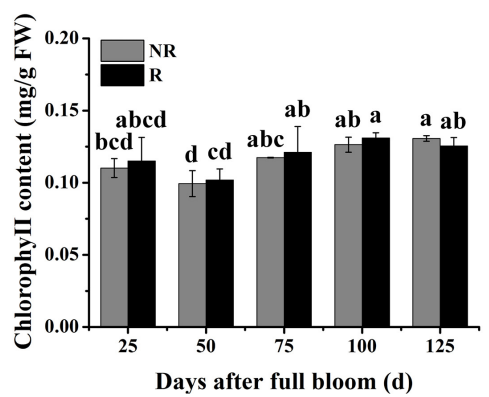

C

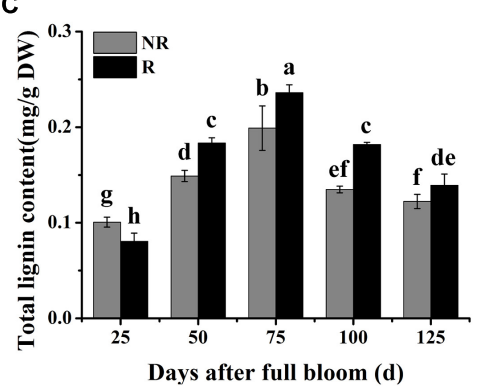

FIGURE 1 | Changes in skin color and pigment contents in F 1 plants from the cross between 'Niitaka' (russet skin pear cultivar, NTK) and 'Dangshansu' (non-russet skin pear cultivar, DSS). (A) Color development in pear skin of $F_{1}$ progeny from 25 to 125 days after full bloom. (B) Chlorophyll and (C) lignin contents in pear skin of $\mathrm{F}_{1}$ progeny from 25 to 125 days after full bloom. NR: non-russet skin fruits; R: russet skin fruits. Different lowercase letters above bars mean significant differences between NR and R groups by Tukey's multiple range tests $(\rho<0.05)$.

GV3101 harboring ProR::PpMYB36 and ProNR::PpMYB36 were transiently transformed into pear skin.

\section{Statistical Analysis}

All experiments were repeated three times. SPSS 22.0 (IBM Corp., Armonk, NY, United States) was used to conduct an analysis of variance (ANOVA) followed by Fisher's least significant difference or Student's $t$-test analysis. Statistically significant differences were tested by Tukey's post hoc tests $(p<0.05)$.

\section{RESULTS}

\section{Phenotypic Evaluation and Color Development in Fruit Skins of $F_{1}$ Pear Plants}

Pear fruits expressing the russet and non-russet skin phenotypes segregated in the $F_{1}$ population obtained from the NTK $\times$ DSS cross. The russet skin fruits gradually turned russet in color after full bloom, whereas the non-russet skin fruits did not change color (Figure 1A). Among the $150 \mathrm{~F}_{1}$ progeny, there were 78 russet and 72 non-russet skin individuals. Based on a chi-square test $\left(\chi^{2}=0.24\right)$, the segregation of the phenotypes fitted a $1: 1$ ratio $(p>0.05)$, indicating the pattern of genetic inheritance of this quality trait.

Furthermore, the lignin and chlorophyll contents in the skins of the two types of fruits from the $\mathrm{F}_{1}$ plants (russet and non-russet) after full bloom were measured. The lignin contents in the russet skin fruits increased and were significantly higher than those in the non-russet skin fruits (Figure 1B). At 75 DAFB, the lignin content in the russet skin fruits was $0.24 \mathrm{mg} \cdot \mathrm{g}^{-1}$, which was approximately 1.20-fold higher than that in the non-russet skin fruits. In addition, mRNA levels of nine structural genes involved in lignin biosynthesis, $P p P A L, P p C 4 H, P p 4 C L, P p C C R$, $P p C A D, P p b H C T, P p C 3 H, P p C C o A O M T$, and $P p C O M T$, were basically higher in the russet skin fruits than in the non-russet skin fruits during the ripening period (Supplementary Figure 1). However, the chlorophyll contents did not significantly differ between the russet- and non-russet skin fruits (Figure 1C). These results suggest that the enhanced russet pigmentation in pear skin can be attributed to lignin accumulation.

\section{Locating the Major Scaffolds to Linkage Groups Using BSA-Seq Analysis}

After trimming and adapter removal, 352,649,438 paired-end clean reads from Illumina high-throughput sequencing were mapped to the pear genome (Supplementary Table 1). Small variant calling for the datasets and subsequent variant filtering generated 3,071,265, 3,591,714, 4,337,289, and 4,163,476 variants (SNPs and Indels) for DSS, NTK, the non-russet skin fruit bulk (B1), and the russet skin fruit bulk (B2), respectively, that were uniformly distributed throughout the genome (Supplementary Table 2). G' value association algorithms mapped the locus to the NW_008988425.1 and NW_008988489.1 scaffolds (Figure 2A), both of which are located on pear chromosome 8. Venn diagrams of all variants in the skin samples of 


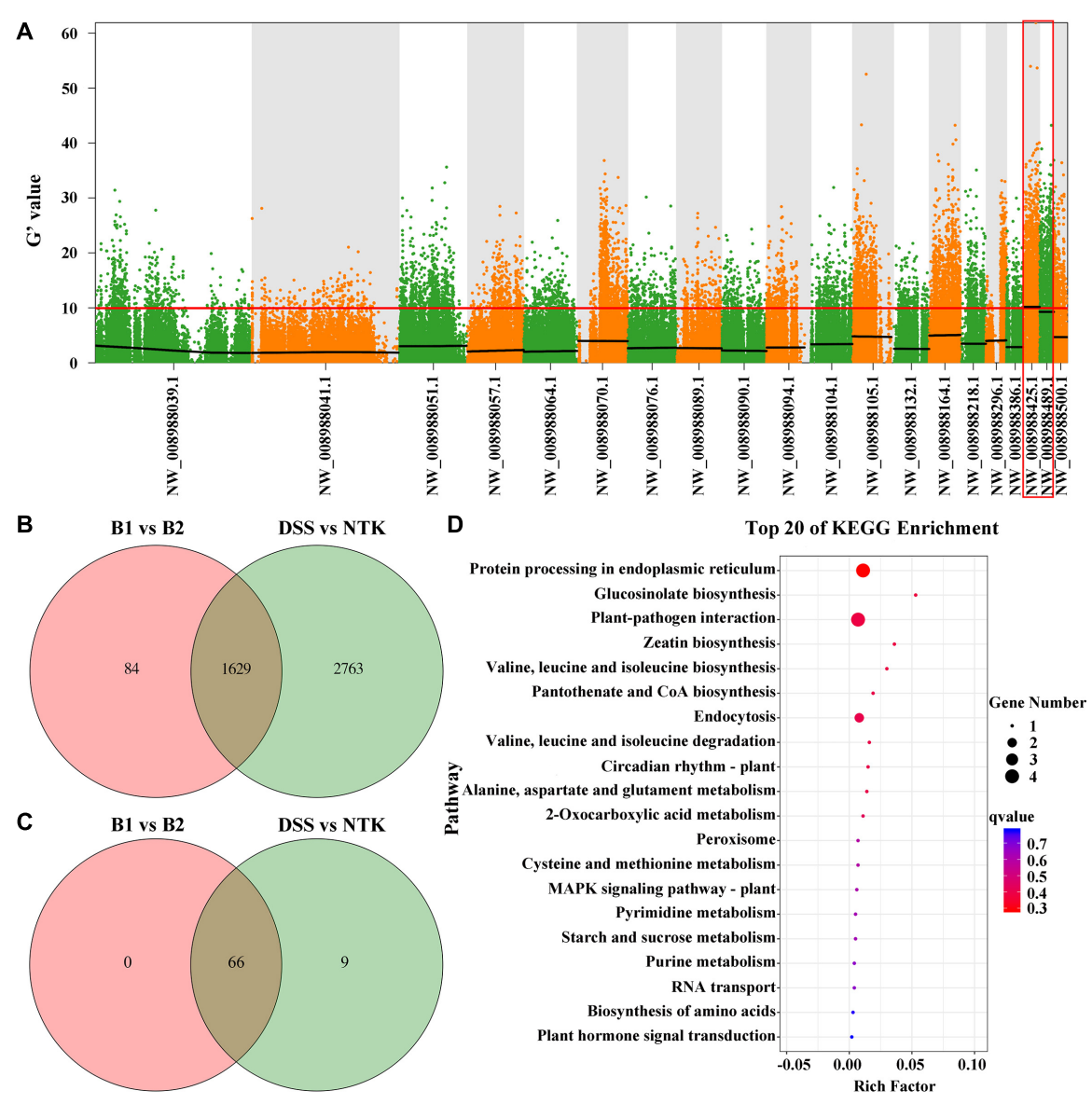

FIGURE 2 | Russet skin trait locus and variant genes identified by bulked segregant analysis combined with the genome sequencing analysis. (A) Using the G' algorithm to map the PpRus gene locus. The distribution of G' values on the chromosome is shown. The abscissa gives the chromosome scaffold names. The colored dots represent the G' values at each single nucleotide polymorphism locus. The red line represents the threshold of significant association. The higher the G' value, the better is the correlation. (B) Venn diagram showing the intersection of all variant genes identified in pairwise analyses (B1 vs. B2 and DSS vs. NTK). DSS: 'Dangshansu' (non-russet skin pear cultivar); NTK: 'Niitaka' (russet skin pear cultivar); B1: non-russet skin fruit bulk; B2: russet skin fruit bulk. (C) Venn diagram representation of variant genes from a pairwise comparison of the two scaffolds NW_008988425.1 and NW_008988489.1. (D) KEGG pathway enrichment analysis of variant genes in the scaffolds NW_008988425.1 and NW_008988489.1.

the B1 vs. B2 and DSS vs. NTK comparisons are shown in Figure 2B. Sixty-six variant genes in the intersection of the Venn diagram in NW_008988425.1 and NW_008988489.1 were also identified (Figure 2C). The significantly enriched KEGG pathways were related to protein processing in the endoplasmic reticulum, glucosinolate biosynthesis, and plant-pathogen interaction (Figure 2D).

\section{Simple Sequence Repeat Markers and Genetic Linkage Map of the PpRus \\ Locus}

On the basis of the BSA-seq results, approximately 50 SSR primer pairs designed from sequences of NW_008988425.1 and NW_008988489.1 were analyzed (Supplementary Table 3). By screening these primer pairs on the $\mathrm{F}_{1}$ progeny (150 plants) from the NTK $\times$ DSS cross, two SSR markers (PpSSRa19 and PpSSRa60) were found to be linked to the PpRus locus. The amplification profiles of PpSSRa19 and PpSSRa60 are shown in Figure 3A. Linkage analysis revealed that the
PpRus locus is flanked by the PpSSRa19 and PpSSRa60 loci, both of which were the nearest marker loci to the PpRus locus, with genetic distances of 8.3 and $15.5 \mathrm{cM}$, respectively (Figure 3B). Both of these marker loci flanking $P p R u s$ were located on the same scaffold (NW_008988489.1), which means that PpRus maps to scaffold NW_008988489.1 (53297211921) in the pear genome (Figure 3C). There were eight genes (LOC103929635, LOC103929636, LOC103929637, LOC103929638, LOC103929640, LOC103929641, LOC103929642, and LOC103929643) among the 66 variant genes (Figure 2C) identified within the region between the marker loci PpSSRa19 and PpSSRa60 (Figure 3D).

\section{Expression Patterns of the Candidate Genes for Russet Skin Color in Pear Fruits}

To determine whether the predicted genes are involved in the trait differences between russet and non-russet skinned fruits, we quantified the expression of the eight candidate 


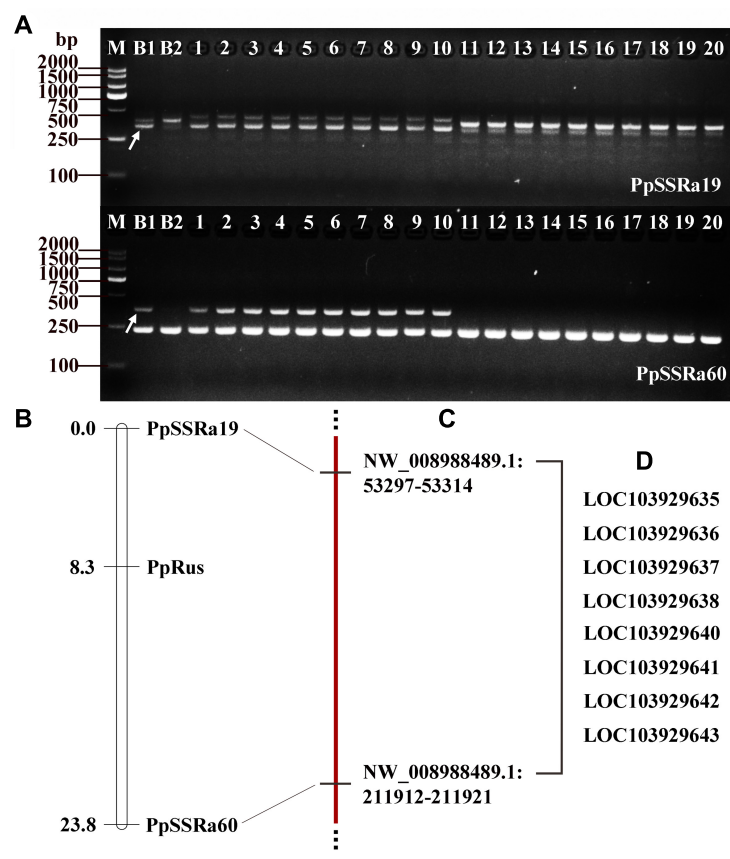

FIGURE 3 | Simple sequence repeat (SSR) markers and genetic linkage mapping of the PpRus locus. (A) Two SSR markers co-segregate with PpRus. M: DNA size marker DL2000; B1: non-russet skin fruit bulk; B2: russet skin fruit bulk; 1-10: non-russet skin individuals; 11-20: russet skin individuals. The polymorphic DNA fragments are indicated by arrows. (B) Genetic linkage map around the PpRus locus created from a segregating $F_{1}$ progeny population of 150 plants from the cross between 'Niitaka' (russet skin pear cultivar) and 'Dangshansu' (non-russet skin pear cultivar). (C) The physical positions of SSR marker loci linked to the PpRus locus in the pear genome. (D) The eight predicted genes within the region flanked by the two marker loci that are the most tightly linked to PpRus.

genes (Figure 3D) by qRT-PCR. The overall trends of gene transcription in the russet skin fruits showed that the genes were up-regulated (Figure 4). The LOC103929635, LOC103929636, LOC103929637, LOC103929638, and LOC103929642 transcript levels in the russet skin fruits reached peak levels at 50 DAFB, and were 1. 25-, 47. 1-, 1. 16-, and 1.43-fold higher, respectively, compared to the genes in the non-russet skin fruits. Additionally, the expression of LOC103929640, LOC103929641, and LOC103929643 reached their highest levels at 75 DAFB, and were higher by 1. 39-, 1. 84-, and 1.31-fold, respectively, in the russet skin fruits compared to the non-russet skin fruits. Interestingly, the LOC103929640 transcript levels followed almost the same trend as the lignin contents in the russet- and non-russet fruit skins (Figures 1C, 4).

\section{LOC103929640 Is Orthologous to the AtMYB36 Transcription Factor From Arabidopsis thaliana}

The coding sequences of LOC103929640 were cloned from both russet and non-russet skin pears. There were no differences in the amino acid sequences of the LOC103929640 proteins between the two different fruit types (Figure 5A). To further analyze the function of LOC103929640, we performed BLAST analysis using the amino acid sequence of LOC103929640 as a search query. The result showed that LOC103929640 belongs to a MYB domain-containing protein family. Moreover, to determine the similarity and relationship of the LOC103929640 sequence to the MYBs of Arabidopsis, we constructed a phylogenetic tree based on an alignment of the amino acid sequences of 25 AtMYBs. The result indicated that LOC103929640 is most closely related to AtMYB36 (Figure 5B). Therefore, we named LOC103929640 PpMYB36. In addition, to determine the subcellular location of PpMYB36, we transiently expressed PpMYB36 fused to green fluorescence protein (GFP) in $N$. benthamiana leaf cells and observed that the PpMYB36-GFP fusion protein localized to the nucleus (Figure 5C).

\section{Phenotypes of PpMYB36-Overexpressing and RNAi-Silenced Pear Fruit Skins}

To investigate the function of PpMYB36 in the regulation of russet fruit skin coloration in pear, the constructs 35S::PpMYB36 and 35S::anti-PpMYB36 were introduced into pear skins by agroinfiltration. Fruits infiltrated with 35S::PpMYB36 displayed enhanced russet pigmentation around the injection sites, while 35S::GUS (control) and 35S::anti-PpMYB36 fruits basically did not change color (Figure 6A). The $P p M Y B 36$ transcription level and lignin content in pear skin expressing with $355:: P$ PMYB36 was significantly higher than in the control and skin infiltrated with 35S::anti-PpMYB36 (Figures 6B,C). Furthermore, the expression of nine structural genes, $P b P A L, P b C 4 H, P b 4 C L$, PbCCR, $\mathrm{PbCAD}, \mathrm{PbHCT}, \mathrm{PbC} 3 \mathrm{H}, \mathrm{PbCCoAOMT}$, and PbCOMT, that are involved in lignin biosynthesis, showed significantly higher levels in pear skin infiltrated with 35S::PpMYB36 compared to the control and 35S::anti-PpMYB36 infiltrated skins at 6 and 12 days after treatment (Supplementary Figure 2). These results indicate that $P$ PMYB36 is responsible for the increased accumulation of lignin and russet coloration in pear skin.

\section{Cloning and Activity Analysis of the Promoter Region of PpMYB36}

We characterized the PpMYB36 upstream regions in order to determine whether the sequence polymorphisms could possibly explain the different coloration patterns in the skins of the two types of pear. Genomic DNA fragments encompassing approximately $1.8 \mathrm{~kb}$ of the promoter region were isolated from both russet and non-russet skin pears. We found a W-box element (-904 bp) insertion in the promoter of PpMYB36 in russet skin pears but not in the $P$ PMYB36 promoter in nonrusset skin pears (Figure 7A). To evaluate the relationship between the different PpMYB36 promoter sequences and gene expression levels in russet skin and non-russet skin fruits, we first performed a dual-LUC reporter assay. ProR and ProNR were cloned into the corresponding sites of $p B 1121$. LUC under the control of the Super promoter was the internal control for infiltration efficiency. Three days after transforming the genes into callus, the GUS and LUC activities were detected, and the GUS/LUC ratio of ProR was significantly higher 


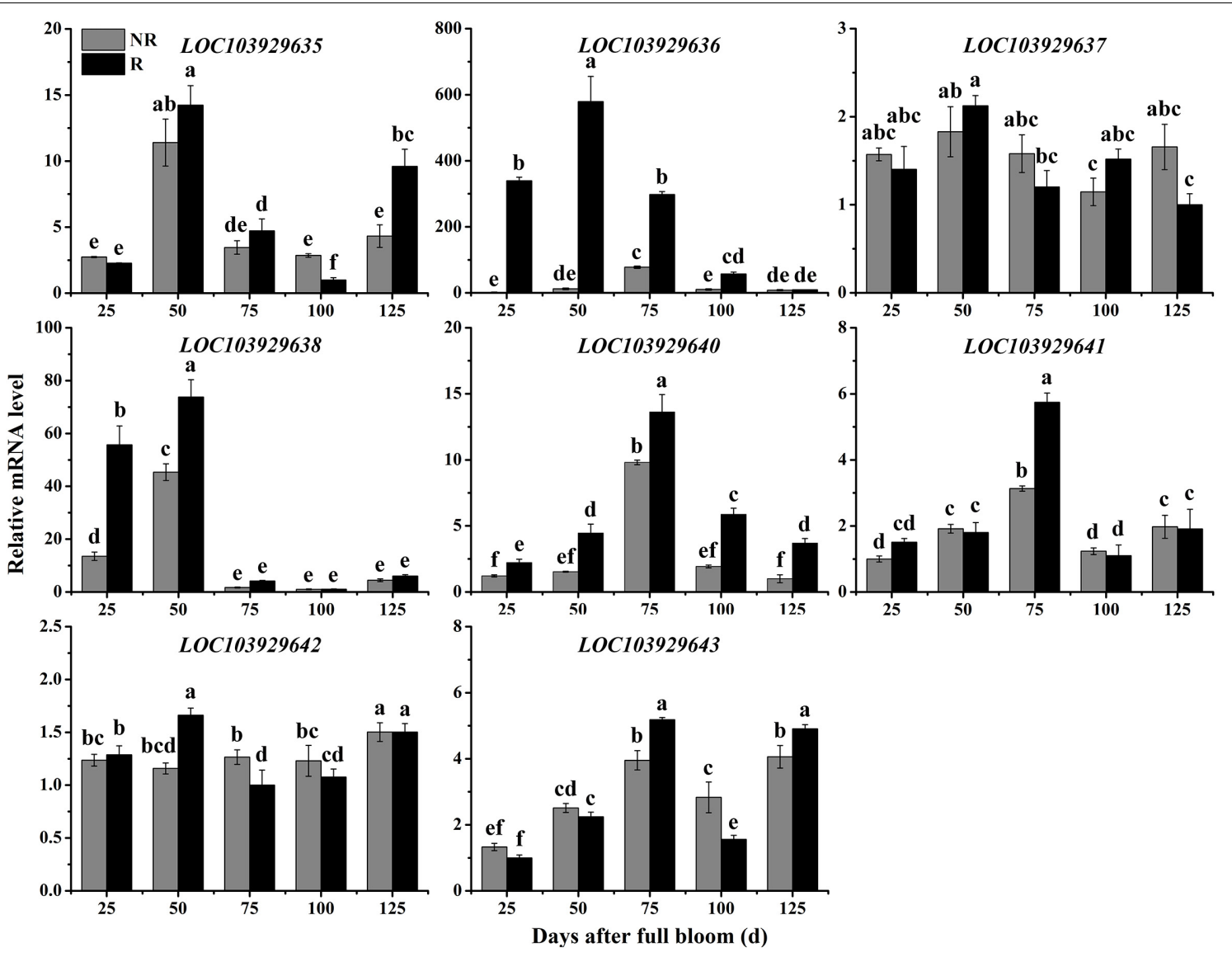

FIGURE 4 | Relative expression levels of LOC103929635, LOC103929636, LOC103929637, LOC103929638, LOC103929640, LOC103929641, LOC103929642, and LOC103929643 in fruit skins of $F_{1}$ progeny from the cross between 'Niitaka' (russet skin pear cultivar, NTK) and 'Dangshansu' (non-russet skin pear cultivar, DSS). NR: non-russet skin fruits; R: russet skin fruits. Different lowercase letters above bars mean significant differences between NR and R groups by Tukey's multiple range tests $(p<0.05)$.

than that of ProNR (Figure 7B). To test the function of the PpMYB36 promoter, we constructed ProR::PpMYB36 and ProNR::PpMYB36, which were then transiently expressed in pear skin. Fruits infiltrated with ProR::PpMYB36 displayed enhanced russet coloration compared with fruits infiltrated with ProNR::PpMYB36 (Figure 7C). Additionally, PpMYB36 expression and lignin content, which were driven by $\operatorname{ProR}$, were significantly higher than when driven by ProNR (Figures 7D,E). These results suggest that transcription of $P$ PMYB36 and lignin accumulation in the russet skin pear are influenced by the promoter sequence.

\section{DISCUSSION}

Russet skin is a vital trait affecting both fruit quality and stress tolerance in pear. Although several studies on russet pear have enabled a better comprehension of the mechanical causes responsible for this phenomenon (Heng et al., 2016; Shi et al., 2019b), the genetic and molecular mechanisms underpinning russet skin coloration have not been thoroughly investigated.

\section{Genetic Mapping of the PpRus Locus That Determines the Russet Skin Trait in Pear}

In this study, the field phenotypes determined by visual observation of the NTK $\times$ DSS hybrids during the ripening period showed that the segregation of russet and non-russet skin fruits fitted the hypothesis of a single major gene controlling the trait. Previously, we used $121 \mathrm{~F}_{1}$ pear trees from the cross of 'Whangkeumbae' $\times$ DSS for marker screening and PpRus mapping; the gene locus that determines the fruit russet skin trait was localized to LG8 in pear (Song et al., 2010). Here, using $150 \mathrm{~F}_{1}$ pear trees from the NTK $\times$ DSS cross, we found that the PpRus locus was located in two scaffolds, NW_008988425.1 and NW_008988489.1 on chromosome 8 of the pear genome (Figure 2A). These results further suggest that the locus associated with the russet skin trait is located on chromosome 8 in pear. Furthermore, pear cultivar breeding is a lengthy process, largely because the trees have long juvenile stages. The MAS approach would improve the efficiency of pear breeding (Kumar et al., 2019; Fiol et al., 2021). The mapped region of the pear genome containing $P p R u s$, which contains 
A

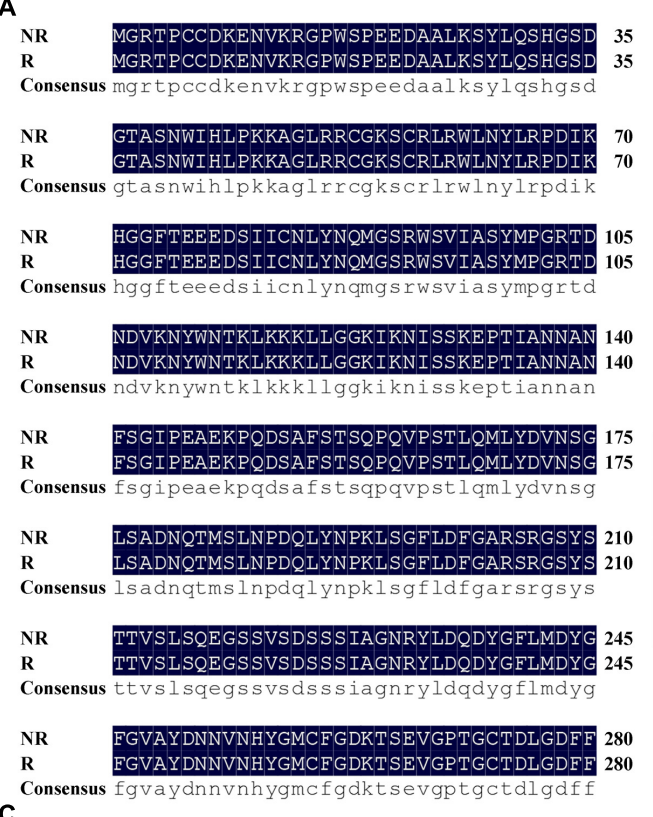

C

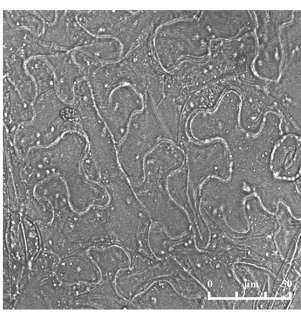

Bright field

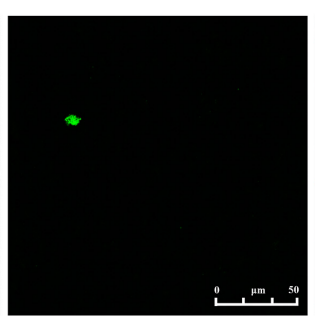

PpMYB36-GFP

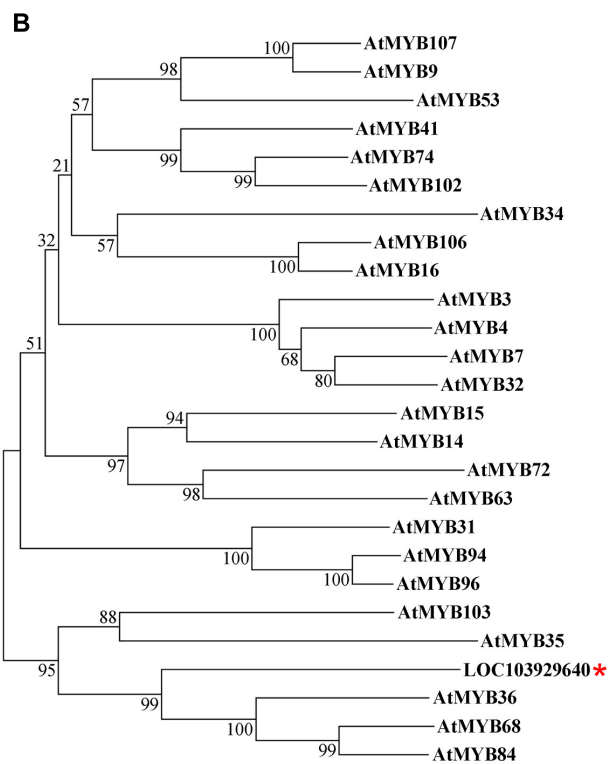

$\longmapsto$

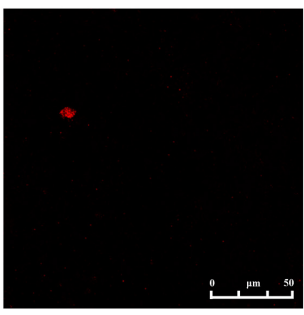

mCherry

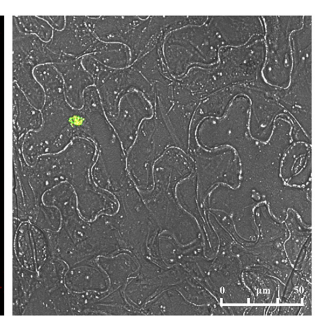

Nuclear merge

FIGURE 5 | Amino acid sequences, phylogenetic tree, and subcellular localization of PpMYB36. (A) Amino acid sequence alignment of the two PpMYB36 proteins from russet (R) and non-russet (NR) pears. (B) A phylogenetic tree showing the relationships between PpMYB36 from pear and 25 MYB protein sequences from Arabidopsis thaliana. Bootstrap values indicate the confidence of each branch. *indicated the position of PpMYB36. (C) Subcellular localization of the PpMYB36-GFP fusion driven by the CaMV35S promoter following transient expression in leaf cells of Nicotiana benthamiana.

two PpRus-linked SSR marker loci, is useful for future pear breeding programs.

\section{Fine Mapping of the PpRus Locus and Identification of a Candidate Gene for the Pear Russet Skin Trait}

Genes involved in the formation of fruit russet skin in pear are clustered into two groups: biosynthesis genes and stressresponsive genes (Legay et al., 2016). No candidate genes have been identified that control the russet skin trait on pear chromosome 8 . Fine mapping of the PpRus locus is critical to the identification of such candidate genes. By identifying marker loci that are tightly linked to and that flank a desired gene locus, the position of the gene can be narrowed down to a small region in the chromosome or contig. Using this strategy, some candidates for the pear $P c D w$ gene have been identified from the most probable region (Wang et al., 2011; Wang C. H. et al., 2016). In the present study, we predicted eight genes as candidates for PpRus in the region between PpSSRa19 and PpSSRa60 (Figure 3D and Supplementary Table 4). This is the first report that identifies a candidate gene possibly associated with russet skin coloration in pear. Notably, among these variant genes, LOC103929640 (PpMYB36) generally exhibited an expression pattern similar to the trend in lignin contents in the two types fruits during the ripening period (Figures 1C, 4). Therefore, we suspect that $P$ PMYB36 plays a crucial role in the russet skin coloration phenotype in pear fruits.

\section{Relationship Between Lignin Accumulation and Russet Skin Coloration in Pear}

Previous studies have shown that lignin biosynthesis can regulate russet skin formation in the russet skin mutant DSS of pear (Heng et al., 2016). Here, we observed considerably higher lignin contents in russet skin fruits than in non-russet skin fruits (Figure 1C), yet chlorophyll contents were not significantly different between the two fruit types (Figure 1B). Accordingly, the change in chlorophyll content may be not the main reason for russet skin coloration in pear fruits; rather, the enhanced russet pigmentation can be mainly attributed to lignin accumulation. 


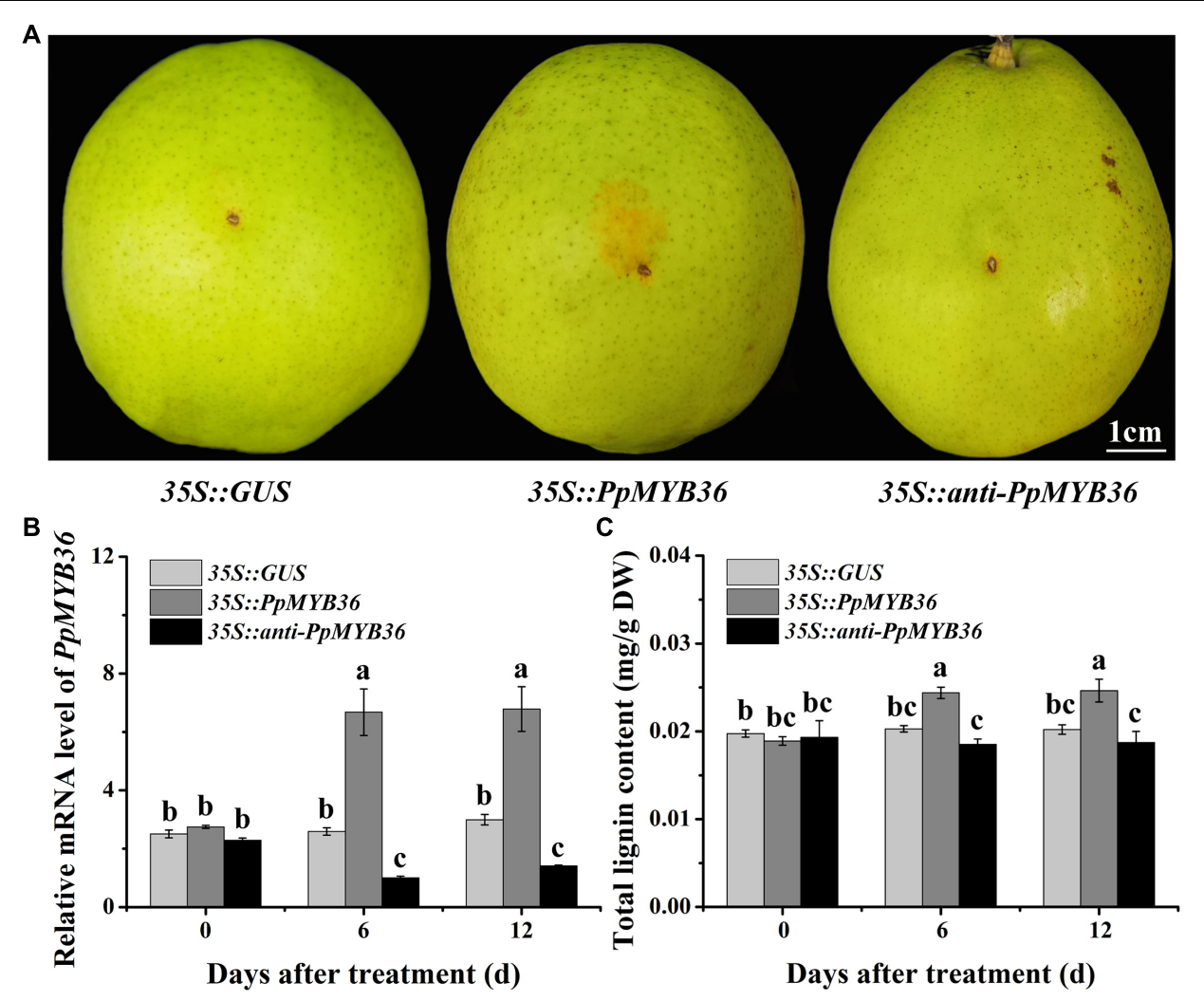

FIGURE 6 | Overexpression or silencing of the PpMYB36 gene in pear fruit skin. (A) Skin phenotypes of infiltrated fruits. (B) Relative expression levels of PpMYB36 and $\mathbf{( C )}$ concentration of lignin in the skins of infiltrated fruits. Different lowercase letters above bars mean significant differences among the treatments by Tukey's multiple range tests $(p<0.05)$.

These results are similar to observations in apple (Malus pumila Mill.) and grape (Vitis vinifera) (Legay et al., 2016; Huang et al., 2020). In addition, the crucial genes involved in lignin biosynthesis have been shown to participate in russet skin coloration in pear (Wang et al., 2014; Zhang et al., 2021). In the present study, we observed much higher expression levels of the PpPAL, PpC4H, Pp4CL, PpCCR, PpCAD, PpHCT, PpC3H, $P p C C o A O M T$, and $P p C O M T$ genes in russet skin fruits than in non-russet skin fruits (Supplementary Figure 1), which also suggests the indispensable role of lignin accumulation in russet skin coloration in pear fruits. Hence, the mechanisms that regulate lignin biosynthesis, which in turn leads to coloration differences in pear fruit skins, require further research.

\section{PpMYB36 Controls the Coloration of Russet Skin Pear}

As essential transcription factors, MYBs are involved in vital regulatory networks that regulate plant development, responses to biotic and abiotic stresses, and lignin biosynthesis (Dubos et al., 2010; An et al., 2019). A recent study has shown that CsMYB36 is involved in the formation of yellow green peel in cucumber (Cucumis sativus) (Hao et al., 2018). In addition, MdMYB93 regulates suberin deposition in russeted apple fruit skins (Legay et al., 2016). In the present study, russet pigmentation was enhanced while a remarkable increase in lignin accumulation was induced in pear fruits infiltrated with 35S::PpMYB36 (Figure 6). This result suggests that $P$ PMYB36 is crucial for regulation of lignin accumulation and russet coloration in pear. Moreover, the MYB transcription factors MYB20, MYB42, MYB43, and MYB85 are transcriptional regulators that directly activate lignin biosynthesis genes in Arabidopsis (Geng et al., 2020). Here, the expression levels of the nine structural genes involved in lignin biosynthesis substantially increased in pear skin infiltrated with 35S::PpMYB36, and the expression patterns of these genes were basically consistent with $P$ PMYB36 transcription levels after the infiltration treatment (Figure 6B and Supplementary Figure 2). It would be interesting to investigate whether PpMYB36 is involved in the activation of these structural genes that mediate lignin biosynthesis, thereby influencing the russet coloration of fruit skin in pear. Such work will further illustrate the regulatory role of $P$ PMYB36 in the russet skin trait of pear.

\section{Variation in the PpMYB36 Promoter Sequence Affects Lignin Accumulation}

To explore the reasons for the differential expression levels of $P p M Y B 36$ in russet and non-russet skin pears, we compared the deduced protein sequences of PpMYB36 and found no difference between the two types fruits (Figure 5A). Nonetheless, we found 
A

NR
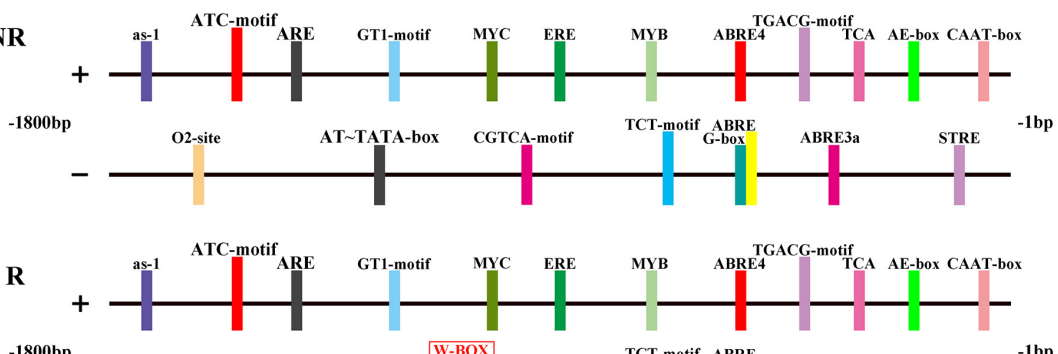

$-1800 \mathrm{bp}$
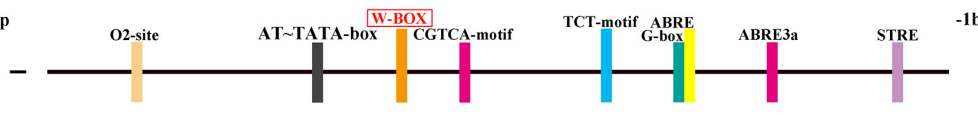

B

C

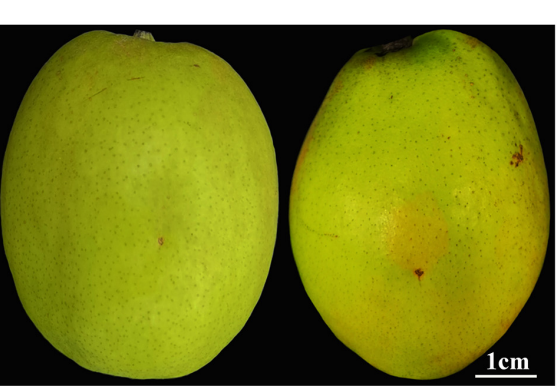

ProNR::PpMYB36

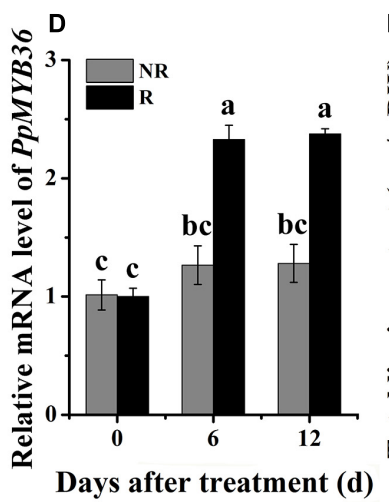

E

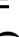
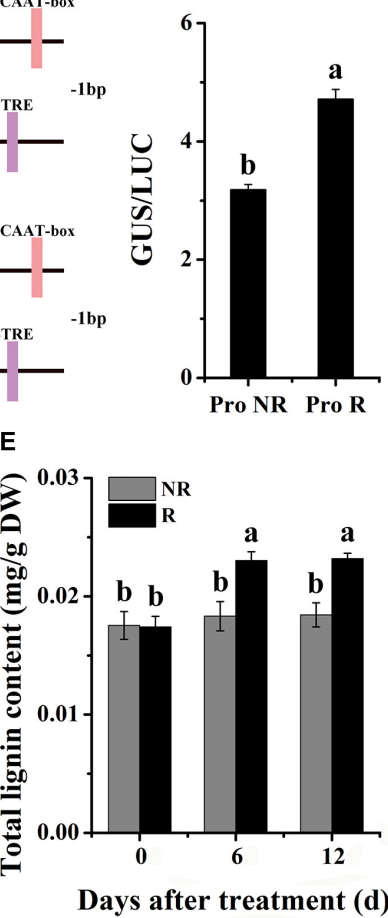

FIGURE 7 | Functional analysis of the PpMYB36 promoter region. (A) Diagrammatic representation of cis-elements present in the PpMYB36 promoter (not drawn to scale). NR: non-russet skin fruits; R: russet skin fruits. (B) Relative GUS/LUC activity in infiltrated callus. ProNR: PpMYB36 promoter from non-russet skin pear; ProR: PpMYB36 promoter from russet skin pear. (C) Phenotypes of pear skins infiltrated with agrobacterium harboring ProNR:PpMYB36 and ProR:PpMYB36. (D) Relative expression levels of PpMYB36 in the skins of infiltrated fruits 0 to 12 days after agroinfiltration. (E) Concentration of lignin in the skins of infiltrated fruits 0 to 12 days after agroinfiltration. Different lowercase letters above bars mean significant differences between NR and R groups by Tukey's multiple range tests $(p<0.05)$.

a W-box (-904 bp) insertion in the promoter sequence of PिMYB36 in the russet skin type compared to the non-russet skin type (Figure 7A; Higo et al., 1999). The results of promoter activity assays indicated that ProR had higher activity than ProNR (Figure 7B). Pear fruits infiltrated with ProR::PpMYB36 showed enhanced russet pigmentation and also a substantial increase in lignin accumulation compared to fruits infiltrated with ProNR::PpMYB36 (Figures 7C,E). Hence, the higher expression levels of $P$ PMYB36 in russet skin pears is due to the W-box insertion in the promoter region. The $\mathrm{W}$-box is a WRKY protein binding element (Xie et al., 2021). Several recent studies have shown that WRKY transcription factors can bind to the promoter regions of $M Y B$ genes that regulate physiological and biochemical functions in plants (Lloyd et al., 2017; Liu et al., 2019). However, whether $P p W R K Y$ activates $P p M Y B 36$ expression by binding to the $\mathrm{W}$-box element remains to be determined. Further studies are required to reveal the mechanisms behind the activities of the different PpMYB36 promoters and their roles in the coloration of russet fruit skin in pear.

\section{CONCLUSION}

By combining BSA-seq analysis and SSR marker identification, we mapped the $P p R u s$ locus that determines the russet fruit skin trait in pear to the scaffold NW_008988489.1: 53297-211921 on chromosome 8 of the pear genome. Eight candidate genes were predicted in the mapped region, among which PpMYB36 was experimentally confirmed to control russet skin coloration in pear. Moreover, a W-box (-904 bp) insertion in the PpMYB36 promoter was found to be essential for $P$ pMYB36-mediated regulation of lignin accumulation and russet coloration in pear skin. This study reveals a novel mechanism for determining russet skin coloration in pear, which is crucial for basic research and breeding applications.

\section{DATA AVAILABILITY STATEMENT}

The datasets presented in this study can be found in online repositories. The names of the repository/repositories and accession number(s) can be found in the article/ Supplementary Material.

\section{AUTHOR CONTRIBUTIONS}

$\mathrm{CM}$ and $\mathrm{CW}$ planned and designed the research. XW, MY, $\mathrm{XZ}, \mathrm{ZS}, \mathrm{XL}$, and YT performed the experiments, conducted the fieldwork, and analyzed the data. CM, XW, and CW wrote the 
manuscript. All authors contributed to the article and approved the submitted version.

\section{FUNDING}

This work was supported by the Funds for Agricultural Variety Improvement Project of Shandong Province (2019LZGC008), the Shandong Provincial Natural Science Foundation, China (ZR2020QC146), the High-level Scientific Research Foundation

\section{REFERENCES}

An, C., Sheng, L., Du, X., Wang, Y., Zhang, Y., Song, A., et al. (2019). Overexpression of $C m M Y B 15$ provides chrysanthemum resistance to aphids by regulating the biosynthesis of lignin. Hortic. Res. 6, 1-10. doi: 10.1038/s41438019-0166-y

Bai, S., Tao, R., Tang, Y., Yin, L., Ma, Y., Ni, J., et al. (2019). BBX16, a B-box protein, positively regulates light-induced anthocyanin accumulation by activating MYB10 in red pear. Plant Biotechnol. J. 17, 1985-1997. doi: 10.1111/pbi.13114

Chezem, W. R., Memon, A., Li, F. S., Weng, J. K., and Clay, N. K. (2017). SG2-Type R2R3-MYB transcription factor MYB15 controls defense-induced lignification and basal immunity in Arabidopsis. Plant Cell 29, 1907-1926. doi: 10.1105/tpc. 16.00954

Dougherty, L., Singh, R., Brown, S., Dardick, C., and Xu, K. (2018). Exploring DNA variant segregation types in pooled genome sequencing enables effective mapping of weeping trait in Malus. J. Exp. Bot. 69, 1499-1516. doi: 10.1093/jxb/ erx490

Dubos, C., Stracke, R., Grotewold, E., Weisshaar, B., Martin, C., and Lepiniec, L. (2010). MYB transcription factors in Arabidopsis. Trends Plant Sci. 15, 573-581. doi: 10.1016/j.tplants.2010.06.005

Fiol, A., García-Gómez, B. E., Jurado-Ruiz, F., Alexiou, K., Howad, W., and Aranzana, M. J. (2021). Characterization of Japanese Plum (Prunus salicina) PsMYB10 alleles reveals structural variation and polymorphisms correlating with fruit skin color. Front. Plant Sci. 12:655267. doi: 10.3389/fpls.2021.655267

Geng, P., Zhang, S., Liu, J., Zhao, C., Wu, J., Cao, Y., et al. (2020). MYB20, MYB42, MYB43, and MYB85 regulate phenylalanine and lignin biosynthesis during secondary cell wall formation. Plant Physiol. 182, 1272-1283. doi: 10.1104/pp. 19.01070

Hao, N., Du, Y., Li, H., Wang, C., Wang, C., Gong, S., et al. (2018). CsMYB36 is involved in the formation of yellow green peel in cucumber (Cucumis sativus L.). Theor. Appl. Genet. 131, 1659-1669. doi: 10.1007/s00122-018-3105-7

Heng, W., Liu, L., Wang, M. D., Jia, B., Liu, P., Ye, Z., et al. (2014). Differentially expressed genes related to the formation of russet fruit skin in a mutant of 'Dangshansuli' pear (Pyrus bretchnederi Rehd.) determined by suppression subtractive hybridization. Euphytica 196, 285-297. doi: 10.1007/s10681-0131032-x

Heng, W., Wang, M. D., Yang, J. Y., Wang, Z. T., Jiang, X. H., and Zhu, L. W. (2016). Relationship between $\mathrm{H}_{2} \mathrm{O}_{2}$ in polyamine metabolism and lignin in the exocarp of a russet mutant of 'Dangshansuli' pear (Pyrus bretschneideri Rehd.). Plant Mol. Biol. Rep. 34, 1056-1063. doi: 10.1007/s11105-016-0985-z

Higo, K., Ugawa, Y., Iwamoto, M., and Korenaga, T. (1999). Plant cis-acting regulatory DNA elements (PLACE) database: 1999. Nucleic Acids Res. 27, 297-300. doi: 10.1093/nar/27.1.297

Huang, Y., Liang, D., Xia, H., Lin, L. J., Wang, J., and Lv, X. L. (2020). Lignin and quercetin synthesis underlies berry russeting in 'sunshine muscat' grape. Biomolecules 10:690. doi: 10.3390/biom10050690

Huo, H., Henry, I. M., Coppoolse, E. R., Verhoef-Post, M., Schut, J. W., de Rooij, H., et al. (2016). Rapid identification of lettuce seed germination mutants by bulked segregant analysis and whole genome sequencing. Plant J. 88, 345-360. doi: $10.1111 /$ tpj.13267

Imerovski, I., Dedić, B., Cvejić, S., Miladinović, D., Jocić, S., Owens, G. L., et al. (2019). BSA-seq mapping reveals major QTL for broomrape resistance in four sunflower lines. Mol. Breeding 39, 1-15. doi: 10.1007/s11032-0190948-9 of Qingdao Agricultural University (Grant 663/1121043), and the Breeding Plan of Shandong Provincial Qingchuang Research Team (2019).

\section{SUPPLEMENTARY MATERIAL}

The Supplementary Material for this article can be found online at: https://www.frontiersin.org/articles/10.3389/fpls.2021. 776816/full\#supplementary-material

Inoue, E., Kasumi, M., Sakuma, F., Anzai, H., Amano, K., and Hara, H. (2006). Identification of RAPD marker linked to fruit skin color in Japanese pear (Pyrus pyrifolia Nakai.). Sci. Hortic. 107, 254-258. doi: 10.1016/j.scienta.2005.07.009

Jia, D., Shen, F., Wang, Y., Wu, T., Xu, X., and Zhang, X. (2018). Apple fruit acidity is genetically diversified by natural variations in three hierarchical epistatic genes: MdSAUR37, MdPP2CH and MdALMTII. Plant J. 95, 427-443. doi: 10. $1111 /$ tpj.13957

Kumar, S., Kirk, C., Deng, C. H., Wiedow, C., Qin, M., Espley, R., et al. (2019). Fine-mapping and validation of the genomic region underpinning pear red skin colour. Hortic. Res. 6, 1-7. doi: 10.1038/s41438-018-0112-4

Lam, P. Y., Tobimatsu, Y., Takeda, Y., Suzuki, S., Yamamura, M., Umezawa, T., et al. (2017). Disrupting flavone synthase II alters lignin and improves biomass digestibility. Plant Physiol. 174, 972-985. doi: 10.1104/pp.16.01973

Lee, S. B., Eun, K. J., Kim, H. T., Gyu-Myung, L., Byung-Soo, K., and Min, L. J. (2020). Genetic mapping of the $c 1$ locus by GBS-based BSA-seq revealed pseudoresponse regulator 2 as a candidate gene controlling pepper fruit color. Theor. Appl. Genet. 133, 1897-1910. doi: 10.1007/s00122-020-03565-5

Legay, S., Guerriero, G., André, C., Guignard, C., Cocco, E., Charton, S., et al. (2016). MdMyb93 is a regulator of suberin deposition in russeted apple fruit skins. New Phytol. 212, 977-991. doi: 10.1111/nph.14170

Legay, S., Guerriero, G., Deleruelle, A., Lateur, M., Evers, D., André, C. M., et al. (2015). Apple russeting as seen through the RNA-seq lens: strong alterations in the exocarp cell wall. Plant Mol. Biol. 88, 21-40. doi: 10.1007/s11103-0150303-4

Li, H., and Durbin, R. (2009). Fast and accurate short read alignment with BurrowsWheeler transform. Bioinformatics 25, 1754-1760. doi: 10.1093/bioinformatics/ btp324

Li, H., Handsaker, B., Wysoker, A., Fennell, T., Ruan, J., Homer, N., et al. (2009). The sequence alignment/map format and SAMtools. Bioinformatics 25, 20782079. doi: 10.1093/bioinformatics/btp352

Lichtenthaler, H. K., and Wellburn, A. R. (1983). Determinations of total carotenoids and chlorophylls $a$ and $b$ of leaf extracts in different solvents. Biochem. Soc. Trans. 11, 591-592. doi: 10.1042/bst0110591

Liu, Q., Luo, L., and Zheng, L. (2018). Lignins: biosynthesis and biological functions in plants. Int. J. Mol. Sci. 19:335. doi: 10.3390/ijms19020335

Liu, W., Wang, Y., Yu, L., Jiang, H., Guo, Z., Xu, H., et al. (2019). MdWRKY11 participates in anthocyanin accumulation in red-fleshed apples by affecting MYB transcription factors and the photoresponse factor MdHY5. J. Agric. Food Chem. 67, 8783-8793. doi: 10.1021/acs.jafc.9b02920

Livak, K. J., and Schmittgen, T. D. (2001). Analysis of relative gene expression data using real-time quantitative PCR and the $2^{-\Delta \Delta C T}$ method. Methods 25 , 402-408. doi: 10.1006/meth.2001.1262

Lloyd, A., Brockman, A., Aguirre, L., Campbell, A., Bean, A., Cantero, A., et al. (2017). Advances in the MYB-bHLH-WD repeat (MBW) pigment regulatory model: addition of a WRKY factor and co-option of an anthocyanin MYB for betalain regulation. Plant Cell Physiol. 58, 1431-1441. doi: 10.1093/pcp/pcx075

Ma, C., Jing, C., Chang, B., Yan, J., Liang, B., Liu, L., et al. (2018a). The effect of promoter methylation on $M d M Y B 1$ expression determines the level of anthocyanin accumulation in skins of two non-red apple cultivars. BMC Plant Biol. 18:108. doi: 10.1186/s12870-018-1320-7

Ma, C., Liang, B., Chang, B., Liu, L., Yan, J., Yang, Y., et al. (2018b). Transcriptome profiling reveals transcriptional regulation by DNA methyltransferase inhibitor 5-Aza-2'-deoxycytidine enhancing red pigmentation in bagged 'Granny Smith' apples (Malus domestica). Int. J. Mol. Sci. 19:3133. doi: 10.3390/ijms19103133 
Ma, C., Liang, B., Chang, B., Yan, J., Liu, L., Wang, Y., et al. (2019). Transcriptome profiling of anthocyanin biosynthesis in the peel of 'granny smith' apples (Malus domestica) after bag removal. BMC Genomics 20:353. doi: 10.1186/s12864-0195730- 1

Magwene, P. M., Willis, J. H., and Kelly, J. K. (2011). The statistics of bulk segregant analysis using next generation sequencing. PLoS Comput. Biol. 7:e1002255. doi: 10.1371/journal.pcbi.1002255

Murashige, T., and Skoog, F. (1962). A revised medium for rapid growth and bio assays with tobacco tissue cultures. Physiol. Plantarum 15, 473-497. doi: 10.1111/j.1399-3054.1962.tb08052.x

Ohtani, M., and Demura, T. (2019). The quest for transcriptional hubs of lignin biosynthesis: beyond the NAC-MYB-gene regulatory network model. Curr. Opin. Biotech. 56, 82-87. doi: 10.1016/j.copbio.2018 .10 .002

Shi, C. H., Qi, B., Wang, X. Q., Shen, L. Y., Luo, J., and Zhang, Y. X. (2019a). Proteomic analysis of the key mechanism of exocarp russet pigmentation of semi-russet pear under rainwater condition. Sci. Hortic. 254, 178-186. doi: 10.1016/j.scienta.2019.04.086

Shi, C. H., Wang, X. Q., Zhang, X. Y., Shen, L. Y., Luo, J., and Zhang, Y. X. (2019b). Response of fruit bagging to lignin biosynthesis and expression of related genes in fruit peel of sand pear (Pyrus pyrifolia Nakai.) cv. Cuiguan. Hortscience 54, 1989-1997. doi: 10.21273/HORTSCI14382-19

Shi, D., Wu, J., Tang, H., Yin, H., Wang, H., Wang, R., et al. (2019c). Single-pollen-cell sequencing for gamete-based phased diploid genome assembly in plants. Genome Res. 29, 1889-1899. doi: 10.1101/gr.2510 33.119

Song, W., Wang, C. H., Tian, Y. K., Tian, W., and Yin, H. (2010). SSR molecular markers linked to the fruit russet skin of pear. Acta Hortic. Sin. 37, 1325-1328. doi: 10.16420/j.issn.0513-353x.2010 .08 .007

Tamura, K., Peterson, D., Peterson, N., Stecher, G., Nei, M., and Kumar, S. (2011). MEGA5: molecular evolutionary genetics analysis using maximum likelihood, evolutionary distance, and maximum parsimony methods. Mol. Biol. Evol. 28, 2731-2739. doi: 10.1093/molbev/msr121

Terakami, S., Shoda, M., Adachi, Y., Gonai, T., Kasumi, M., Sawamura, Y., et al. (2006). Genetic mapping of the pear scab resistance gene Vnk of Japanese pear cultivar Kinchaku. Theor. Appl. Genet. 113, 743-752. doi: 10.1007/s00122-0060344-9

Wang, C., Tian, Y., Buck, E. J., Gardiner, S. E., Dai, H., and Jia, Y. (2011). Genetic mapping of PcDw determining pear dwarf trait. J. Am. Soc. Hortic. Sci. 136, 48-53. doi: 10.21273/JASHS.136.1.48

Wang, C. H., Li, W., Tian, Y. K., Hou, D. L., and Bai, M. D. (2016). Development of molecular markers for genetic and physical mapping of the $\mathrm{PcDw}$ locus in pear (Pyrus communis L.). J. Hortic. Sci. Biotech. 91, 299-307. doi: 10.1080/14620316. 2016.1155319

Wang, Y. Z., Dai, M. S., Cai, D. Y., Zhang, S., and Shi, Z. B. (2016). A review for the molecular research of russet/semi-russet of sand pear exocarp and their genetic characters. Sci. Hortic. 210, 138-142. doi: 10.1016/j.scienta.2016 .07 .019
Wang, Y. Z., Dai, M. S., Zhang, S. J., and Shi, Z. B. (2014). Exploring candidate genes for pericarp russet pigmentation of sand pear (Pyrus pyrifolia) via RNASeq data in two genotypes contrasting for pericarp color. PLoS One 9:e83675. doi: 10.1371/journal.pone.0083675

Wang, Z., Du, H., Zhai, R., Song, L., Ma, F., and Xu, L. (2017). Transcriptome analysis reveals candidate genes related to color fading of 'Red Bartlett' (Pyrus communis L.). Front. Plant Sci. 8:455. doi: 10.3389/fpls.2017.00455

Wu, J., Wang, Z., Shi, Z., Zhang, S., Ming, R., Zhu, S., et al. (2013). The genome of the pear (Pyrus bretschneideri Rehd.). Genome Res. 23, 396-408. doi: 10.1101/ gr.144311.112

Xie, W., Ke, Y., Cao, J., Wang, S., and Yuan, M. (2021). Knock out of transcription factor WRKY53 thickens sclerenchyma cell walls, confers bacterial blight resistance. Plant Physiol. 1-16. doi: 10.1093/plphys/kiab400

Xu, J., Zhang, Y., Qi, D., Huo, H., Dong, X., Tian, L., et al. (2021). Metabolomic and transcriptomic analyses highlight the influence of lipid changes on the post-harvest softening of Pyrus ussurian Max. 'zaoshu shanli'. Genomics 113, 919-926. doi: 10.1016/j.ygeno.2020.10.025

Yamamoto, T., Terakami, S., Takada, N., Nishio, S., Onoue, N., Nishitani, C., et al. (2014). Identification of QTLs controlling harvest time and fruit skin color in Japanese pear (Pyrus pyrifolia Nakai.). Breeding Sci. 64, 351-361. doi: $10.1270 /$ jsbbs.64.351

Zhang, M. Y., Xue, C., Hu, H., Li, J., Xue, Y., Wang, R., et al. (2021). Genome-wide association studies provide insights into the genetic determination of fruit traits of pear. Nat. Commun. 12, 1-10. doi: 10.1038/s41438-020-0242-3

Zhang, S., Feng, M., Chen, W., Zhou, X., Lu, J., Wang, Y., et al. (2019). In rose, transcription factor PTM balances growth and drought survival via PIP2; 1 aquaporin. Nat. Plants 5, 290-299. doi: 10.1038/s41477-019-0376-1

Zhao, S., Zhang, M. L., Ma, T. L., and Wang, Y. (2016). Phosphorylation of ARF2 relieves its repression of transcription of the $\mathrm{K}^{+}$transporter gene HAK5 in response to low potassium stress. Plant Cell 28, 3005-3019. doi: 10.1105/tpc. 16.00684

Conflict of Interest: The authors declare that the research was conducted in the absence of any commercial or financial relationships that could be construed as a potential conflict of interest.

Publisher's Note: All claims expressed in this article are solely those of the authors and do not necessarily represent those of their affiliated organizations, or those of the publisher, the editors and the reviewers. Any product that may be evaluated in this article, or claim that may be made by its manufacturer, is not guaranteed or endorsed by the publisher.

Copyright (c) $2021 \mathrm{Ma}$, Wang, Yu, Zheng, Sun, Liu, Tian and Wang. This is an open-access article distributed under the terms of the Creative Commons Attribution License (CC BY). The use, distribution or reproduction in other forums is permitted, provided the original author(s) and the copyright owner(s) are credited and that the original publication in this journal is cited, in accordance with accepted academic practice. No use, distribution or reproduction is permitted which does not comply with these terms. 